\title{
Bring the magic back to the bullets
}

\section{Governments must act swiftly and decisively to incentivize antibiotic $R \& D$ and clarify regulations.}

This focus issue on antibiotics illustrates the adage that one sector's poison is another's meat. The pharmaceutical industry, which 20 years ago was bringing to market approximately five new antibiotics every year, now only commercializes one or two at best. And a study carried out two years ago reported only six antibacterials out of $>500$ drugs in late-stage clinical testing in big pharma firms.

In contrast, biotech industry interest in antimicrobials appears to be flourishing. Firms are attracted by the predictive power of preclinical studies of antibiotics (animal models of infection actually work). They realize that clinical trials can be short and endpoints clear. And pharma has bequeathed projects and clinical compounds for in-licensing at bargain prices together with experienced management for seeding new spinouts. The 'nicheness' of the markets that caused pharma's flight from the sector -notwithstanding such exceptions as Astra Zeneca and Wyeth, which supported this issue-represents an opportunity for small firms.

What's more, the demand for antibacterials can only increase. Urbanization and global travel will inevitably intensify, spreading infections more rapidly and imposing greater evolutionary pressure on pathogens. The human population is aging, creating increasing numbers of just the kind of immunocompromised hosts that opportunistic pathogens love. And those generous folks at Homeland Security seem awfully keen to throw money at the threat of microbial terrorism, regardless of reality.

This rosy view is soured, however, by industry statistics on the current state of antibiotic development. With a trickle of new antibacterial agents making it to market each year, and only two new chemical classes of antibiotic-Pfizer's linezolid and Cubist Pharmaceutical's daptomycin-approved in the past 30 years, there's reason to think that the shortfall in new compounds and the emergence of multidrug-resistant bacteria could prove a deadly combination.

According to the Infectious Diseases Society of America, 70\% of hospital-acquired infections in the United States are now resistant to one antibiotic or more. Methicillin-resistant Staphylococcus aureus (MRSA) is being joined by other opportunistic pathogens, such as Clostridium difficile and Acinetobacter baumannii. In the United States, two million hospitalized patients contract infections each year, at a cost of $\$ 5$ billion annually in extended stays and expensive drug regimens. Around 90,000 of them die from hospital-acquired infections untreatable by antibiotics.

Antibiotic-resistance needs to be tackled by preventive measures. And it needs to be tackled through incentives for antibiotic development.

Governments are - at last—starting to come to grips with the first problem. In October, for instance, the US Centers for Disease Control and Prevention (CDC) outlined strategies to prevent the spread of drugresistant infections in healthcare facilities. Europe, like the CDC, has its Antimicrobial Resistance Surveillance System, which tracks and detects multidrug-resistant strains as they emerge. And in January the European Union banned the use of all antibiotics from animal feed for growth promotion purposes.
Such measures reduce the burden of antibiotic resistance, but they do not address the declining efficacy of existing antibiotics or the lack of new compounds in development.

Part of the problem is simple economics. Most antibiotics generate only \$200-300 million annually. They cure, rather than treat, patients in a few days and do not have to be prescribed for a lifetime, making them less attractive for investment. Patent lifetimes and clinical 'misuse' leading to resistance combine to restrict a drug's commercial life to 8-10 years. And antibiotics for use against resistant bacteria are often reserved as drugs of last resort, further limiting sales.

As if commercialization were not hard enough, regulatory agencies have also muddied the waters. In 2000, the US Food and Drug Administration (FDA) required novel antibiotics to demonstrate cure rates that were not $<10 \%$ lower than (or 'noninferior to') that of a designated existing antibiotic (the earlier standard was 10-20\%). Then in July 2005, unbeknown to industry, FDA again changed its position, requiring placebo-controlled trials rather than noninferiority trials for certain infections.

The 2000 change caused Cubist's daptomycin to miss its clinical endpoints and forced Wyeth to redesign completely its trial of tigecycline (an antibiotic targeting MRSA), doubling the number of patients from 4,000 to 8,000 . The 2005 change became apparent only this September, when FDA notified Oscient Pharmaceuticals that it would not accept noninferiority studies for gemifloxacin mesylate in acute bacterial sinusitis. One month later, it issued a 'non-approvable' letter to Replidyne for faropenem medoxomil in four community-acquired indications.

The FDA needs to recognize that antibacterial development is now in the hands of inexperienced biotech firms (rather than pharma) — guidance on standards of efficacy and safety must therefore be lucid and communication clear. One way for FDA Commissioner Andrew von Eschenbach to rectify matters would be to prioritize the rapid publication of industry guidances on resistant pathogens, bacterial meningitis and other community-acquired infections.

More public money also needs to be channeled specifically into basic research on antibacterials. By comparison, viruses kill only a fraction as many people as bacterial infections in the United States, yet antiviral research receives many times more funding than antibiotic research.

Governments could help by providing market guarantees-promises to buy antibiotics for multidrug-resistant bacteria, much in the same way as incentives for vaccine development. Or they could remove liability obstacles by providing firms that are developing antibiotics against resistant bacteria with immunity from toxicity lawsuits.

In short, legislators need to step up and start providing leadership and incentives. The alternative is a future without effective antibiotics and catastrophic consequences for public health. Surgery would become more hazardous, immunosuppressive treatments and transplantation untenable and hospitals a life-threatening, rather than a life-enhancing, environment. Antibiotics are not just another product class. 